\section{Avaliação das ações de prevenção da transmissão vertical do HIV e sífilis em maternidades públicas de quatro municípios do Nordeste brasileiro}

\author{
Evaluation of measures to prevent vertical \\ transmission of HIV and syphilis in public maternity \\ hospitals in four municipalities in Northeast Brazil
}

Vilma Costa de Macêdo 1 Adriana Falangola Benjamin Bezerra ${ }^{1}$ Paulo Germano de Frias 2 Carla Lourenço Tavares de Andrade 3
1 Programa Integrado de Pós-graduação em Saúde Coletiva, Universidade Federal de Pernambuco, Recife, Brasil.

2 Instituto de Medicina Integral Professor Fernando Figueira, Recife, Brasil.

${ }^{3}$ Escola Nacional de Saúde Pública Sergio Arouca, Fundação Oswaldo Cruz, Rio de Janeiro, Brasil.

Correspondência V. C. Macêdo Programa Integrado de Pós-graduação em Saúde Coletiva, Universidade Federal de Pernambuco. Rua Irmã Maria David 210, apto. 104, Recife, $P E$ 52061-070, Brasil. vilmacmacedo@hotmail.com

\begin{abstract}
This article evaluates the care provided to women in four public maternity hospitals in Northeast Brazil in relation to interventions adopted by the Projeto Nascer to reduce vertical transmission of HIV and syphilis in 2005. This was a multiple case study including one retrospective and another cross-sectional approach. The logical model for the intervention has five components: prevention, epidemiological surveillance, and pharmaceutical, laboratory, and clinical care. For each component, structure and process indicators were selected. The maternity hospitals were classified as: acceptable, partially implemented, unacceptable, and not implemented. The highest adherence to the standards was in maternity hospital $A$ (62.5\%), classified as partially implemented, and the lowest in maternity hospital C (30.6\%), considered unacceptable. The study highlights the importance of preventive measures, epidemiological surveillance, and pharmaceutical, laboratory, and clinical care to reduce vertical transmission of HIV and syphilis.
\end{abstract}

Health Evaluation; Vertical Disease Transmission; HIV; Syphilis

\section{Introdução}

A qualidade da assistência durante a gestação e parto é um importante determinante da redução de transmissão vertical do HIV e da sífilis. No Brasil, a notificação compulsória de gestantes portadoras dessas infecções é obrigatória desde os anos 2000 e 1986, respectivamente 1,2.

Estudos têm mostrado que existe uma diminuição do número de casos de transmissão vertical do HIV, principalmente, a partir dos resultados do Protocolo 076 do AIDS Clinical Trials Group (ACTG 076) e do acesso à terapia medicamentosa 3,4,5. Apesar disso, as taxas de incidência da infecção permanecem elevadas demonstrando a complexidade do problema e das intervenções susceptíveis de resolvê-la, além de que as ações não estão sendo implementadas com a intensidade necessária para reverter a situação 6 .

A sífilis congênita é causa de morbidade na vida intra-uterina, levando a desfechos negativos da gestação em mais de $50 \%$ dos casos 7 . Segundo a Organização Mundial da Saúde (OMS), nos países subdesenvolvidos, em torno de $10 \%$ a $15 \%$ das gestantes seriam portadoras de sífilis 8 . O Ministério da Saúde do Brasil, em 1997, visando a ajustar as metas de controle nacional, considerou como registro aceitável de eliminação até 1 caso por mil nascidos vivos, entretanto, este padrão ainda não foi alcançado e a doença continua como prioridade a ser trabalhada por todos os municípios 9 . 
Pessoas infectadas pelo HIV apresentam também altas taxas de infecção por outras doenças sexualmente transmissíveis (DST), em conseqüência de fatores comportamentais, biológicos, do estado de imunossupressão, ou mesmo resultar da interação de mais de um fator 10 . Outro aspecto a ser observado é o aumento da transmissão heterossexual, com conseqüente feminilização, principalmente entre as mulheres de baixo nível sócio-econômico e que residem em municípios de pequeno porte 11,12,13,14. Essa nova demanda traz consigo a maternidade em situação de infecção pelo HIV 10 .

Com o intuito de fortalecer as ações de redução da morbimortalidade do HIV e da sífilis congênita por transmissão vertical, o Ministério da Saúde implantou o Projeto Nascer, em 2002, nas maternidades do Sistema Único de Saúde (SUS), localizadas em municípios considerados prioritários e que atendem mais de 500 partos por ano segundo a Portaria $n^{\circ}$. 2.104 GM, de 19 de novembro de 200215.

As ações desenvolvidas pelas maternidades envolvem: capacitação de equipes multiprofissionais no acolhimento, aconselhamento, realização de testes rápidos, manejo clínico de parturientes HIV positivas e crianças expostas, testagem e indicação terapêutica para sífilis, bem como a vigilância epidemiológica e disponibilidade do tratamento farmacológico, estabelecido por meio dos protocolos 16 .

Em Pernambuco, existem 16 maternidades cadastradas no Projeto Nascer sendo oito em Recife, quatro na Região Metropolitana (com exceção de Recife) e quatro no interior do estado 17. Considerando que as ações de monitoramento e de avaliação em saúde se apresentam, ainda, pouco incorporadas às práticas do SUS, em particular no interior do nordeste brasileiro, e que as intervenções são voltadas para redução da transmissão vertical de casos em que o subregistro é maior, este estudo objetivou avaliar a implantação do Projeto Nascer no universo das quatro maternidades localizadas no interior do Estado de Pernambuco, cadastradas no Programa Estadual de DST/AIDS.

\section{Material e métodos}

A estratégia utilizada foi o estudo de casos múltiplos por possuir a capacidade de lidar com uma ampla variedade de evidências, abrangendo a lógica do planejamento, técnicas de coleta e abordagens específicas 18 .

Para análise da intervenção do Projeto Nascer foi construído um modelo lógico tomando como base o manual técnico do projeto 16 , portaria 15 , diretrizes 2 e protocolos de assistência clínica 19 e avaliações sobre o tema 6,20 com o propósito de identificar as atividades desempenhadas, produtos esperados e os possíveis resultados. Com base na análise documental foram definidos os componentes integrantes do modelo, a saber: prevenção, vigilância epidemiológica, assistência farmacêutica, laboratorial e clínica (Tabela 1). A existência do modelo possibilitou o confronto com a realidade empírica da assistência prestada às mulheres na maternidade. Foram excluídas ações relacionadas ao seguimento do caso - dimensão da promoção da saúde com qualidade de vida.

A seleção dos indicadores, realizada a partir do modelo lógico (Tabela 2), considerou o seu poder de discriminação, disponibilidade da informação nas fontes de coleta e a importância da ação baseada nos padrões estabelecidos pelo Ministério da Saúde. Nos indicadores considerados por componentes sem o padrão definiram-se ajustes para operacionalizar a construção de um indicador sintético.

O critério de inclusão das maternidades-casos foi estarem localizadas no interior de Pernambuco, excluídas as da região metropolitana do Recife, e credenciadas para desenvolver o Projeto Nascer segundo informações da Coordenação Estadual de DST/AIDS, em junho de 2005. Quatro maternidades se enquadraram no critério por serem as únicas do interior de Pernambuco a desenvolver as ações do Projeto Nascer, sendo denominadas Maternidade A, Maternidade B, Maternidade C e Maternidade D.

Utilizou-se uma abordagem transversal e outra retrospectiva. A transversal compreendeu entrevistas com os gestores, gerentes setoriais (laboratório e farmácia) e, ainda, a observação dos procedimentos adotados na triagem e no pré-parto, e entrevista com uma amostra de parturientes. Tal procedimento objetivou avaliar a assistência prestada às mulheres que buscaram atendimento nas maternidades-casos. A retrospectiva incluiu uma amostra de mulheres atendidas nas maternidades, além dos prontuários de casos confirmados de HIV e sífilis em 2004.

Os instrumentos utilizados foram os adotados na avaliação do grau de implementação do programa de controle de transmissão vertical do HIV em maternidades do Projeto Nascer 6, coordenado pelo Programa Nacional de DST/AIDS. Pequenos ajustes foram realizados no instrumento considerando os objetivos da avaliação. Para sua adequação utilizou-se técnica de consenso presencial envolvendo um grupo de especialistas na área temática, avaliadores externos, gestores e profissionais de saúde dos serviços. A oficina aconteceu na cidade do Recife, no mês de 
Modelo lógico do Projeto Nascer.

\begin{tabular}{|c|c|c|c|c|}
\hline $\begin{array}{l}\text { Componente de } \\
\text { intervenção }\end{array}$ & Insumo & Atividade & Produto & $\begin{array}{c}\text { Resultado } \\
\text { (curto e médio prazo) }\end{array}$ \\
\hline Prevenção & $\begin{array}{l}\text { - Treinamento de } \\
\text { profissionais de saúde } \\
\text { no Projeto Nascer } \\
\text { - Existência de normas } \\
\text { afixadas em ambiente de } \\
\text { triagem e pré-parto para } \\
\text { prevenção da transmissão } \\
\text { vertical do HIV e sífilis } \\
\text { - Cartão do Pré-Natal }\end{array}$ & $\begin{array}{l}\text { - Treinamento para o manejo } \\
\text { e condutas no atendimento } \\
\text { a gestantes HIV+ e com sífilis } \\
\text { - Aconselhamento sobre a } \\
\text { importância do exame } \\
\text { de HIV e sífilis } \\
\text { - Autorização para realização } \\
\text { do teste rápido } \\
\text { - Instituição da assistência } \\
\text { baseada em protocolos } \\
\text { - Normas afixadas para } \\
\text { prevenção da transmissão } \\
\text { vertical do HIV e sífilis } \\
\text { - Cartão do Pré-Natal afixado } \\
\text { no prontuário }\end{array}$ & $\begin{array}{l}\text { - Profissionais de saúde treinados } \\
\text { - Aconselhamento para realização } \\
\text { do exame de HIV e sífilis } \\
\text { - Autorização e consentimento } \\
\text { por escrito para realização } \\
\text { do teste rápido } \\
\text { - Conhecimento do protocolo } \\
\text { e das ações instituídas pelo } \\
\text { Projeto Nascer, além da } \\
\text { disponibilidade para consulta } \\
\text { em local de trabalho } \\
\text { - Identificação das condutas } \\
\text { adotadas no pré-natal } \\
\text { - Fixação de cartazes sobre as } \\
\text { formas de prevenção da transmissão } \\
\text { vertical do HIV e sífilis }\end{array}$ & $\begin{array}{l}\text { - Qualificação dos profissionais } \\
\text { de saúde nas condutas } \\
\text { normatizadas para atendimento } \\
\text { de gestantes com HIV e sífilis } \\
\text { - Instituição do manejo de } \\
\text { casos baseados em protocolos } \\
\text { do Ministério da Saúde } \\
\text { - Divulgação de ações } \\
\text { preventivas na redução da } \\
\text { transmissão vertical do HIV } \\
\text { e sífilis através de materiais } \\
\text { informativos, afixados no } \\
\text { âmbito das maternidades }\end{array}$ \\
\hline $\begin{array}{l}\text { Vigilância } \\
\text { epidemiológica }\end{array}$ & $\begin{array}{l}\text { - Comissão de controle } \\
\text { de infecção hospitalar } \\
\text { - Comissão de revisão } \\
\text { de prontuário } \\
\text { - Comissão de análise } \\
\text { de óbitos } \\
\text { - Fluxograma de } \\
\text { investigação e manejo } \\
\text { dos casos de HIV e sífilis } \\
\text { - Ficha de notificação e } \\
\text { investigação de sífilis } \\
\text { congênita } \\
\text { - Ficha de investigação } \\
\text { de gestante HIV+ e } \\
\text { crianças expostas }\end{array}$ & $\begin{array}{l}\text { - Desenvolvimento de ações } \\
\text { educativas de caráter individual } \\
\text { e coletivo para redução da } \\
\text { transmissão vertical } \\
\text { do HIV e sífilis } \\
\text { - Investigação e notificação dos } \\
\text { casos positivos de HIV e sífilis } \\
\text { - Investigação e notificação dos } \\
\text { casos de morte fetal após } 20 \\
\text { semanas de gestação de mãe } \\
\text { portadora de sífilis ou com } \\
\text { tratamento inadequado } \\
\text { - Busca ativa de casos em fontes } \\
\text { alternativas (laboratórios, } \\
\text { hospitais, SIH) } \\
\text { - Rastreamento do parceiro } \\
\text { para testagem de sífilis. } \\
\text { - Revisão dos prontuários casos } \\
\text { para verificação das condutas } \\
\text { adotadas }\end{array}$ & $\begin{array}{l}\text { - Orientação sobre as principais } \\
\text { formas de transmissão vertical } \\
\text { do HIV e sífilis } \\
\text { - Número de casos de sífilis em } \\
\text { gestantes, recém-nascido com } \\
\text { sífilis congênita, morte fetal } \\
\text { após } 20 \text { semanas de mãe } \\
\text { portadora de sífilis, gestante } \\
\text { HIV+ e crianças expostas } \\
\text { notificadas e investigadas } \\
\text { - Busca ativa dos casos em } \\
\text { laboratório, hospitais, SIH, } \\
\text { entre outros } \\
\text { - Parceiros testados para sífilis } \\
\text { - Avaliação das condutas instituídas } \\
\text { nos prontuários casos positivos de } \\
\text { HIV e sífilis congênita, identificados }\end{array}$ & $\begin{array}{l}\text { - Estabelecimento da incidência } \\
\text { do agravo na maternidade- } \\
\text { caso } \\
\text { - Institucionalização dos casos } \\
\text { identificados como "evento } \\
\text { sentinela" } \\
\text { - Estabelecimento do } \\
\text { fluxograma de notificação, } \\
\text { conduta e tratamento. } \\
\text { - Garantia de uma comissão } \\
\text { para revisão dos prontuários } \\
\text { e análise dos óbitos }\end{array}$ \\
\hline $\begin{array}{l}\text { Assistência } \\
\text { farmacêutica }\end{array}$ & $\begin{array}{l}\text { - Estrutura física } \\
\text { - Estrado ou estante } \\
\text { - Telas em janelas } \\
\text { - Geladeira } \\
\text { - Termômetro } \\
\text { - Medicamento anti- } \\
\text { retroviral } \\
\text { - Inibidor de lactação } \\
\text { - Fórmula láctea } \\
\text { - Medicamento para sífilis }\end{array}$ & $\begin{array}{l}\text { - Serviços em condições } \\
\text { físicas adequadas } \\
\text { - Equipamento com } \\
\text { armazenamento de medicações } \\
\text { adequadas } \\
\text { - Leitura e registro da } \\
\text { temperatura da geladeira } \\
\text { - Fornecimento de drogas anti- } \\
\text { retrovirais: AZT injetável (frascos), } \\
\text { comprimido, xarope, inibidor de } \\
\text { lactação injetável e comprimido, } \\
\text { fórmula láctea } 1 \text { semestre } \\
\text { - Fornecimento de antibiótico } \\
\text { para sífilis: penicilina benzatina, } \\
\text { procaína e cristalina }\end{array}$ & $\begin{array}{l}\text { - Medicamentos armazenados } \\
\text { em condições adequadas } \\
\text { - Dispensação de medicamentos } \\
\text { anti-retrovirais e penicilina com } \\
\text { validade adequada } \\
\text { - Estabelecimento de controle } \\
\text { de estoques, validade e reposição } \\
\text { de medicamentos }\end{array}$ & $\begin{array}{l}\text { - Garantia de dispensar os } \\
\text { medicamentos prescritos para } \\
\text { redução da transmissão vertical } \\
\text { do HIV e tratamento da sífilis } \\
\text { e sífilis congênita } \\
\text { - Instituição de um fluxo } \\
\text { operacional de entrada, saída } \\
\text { e reposição dos medicamentos } \\
\text { anti-retrovirais e antibióticos }\end{array}$ \\
\hline
\end{tabular}

(continua) 
Tabela 1 (continuação)

\begin{tabular}{|c|c|c|c|c|}
\hline $\begin{array}{l}\text { Componente de } \\
\text { intervenção }\end{array}$ & Insumo & Atividade & Produto & $\begin{array}{c}\text { Resultado } \\
\text { (curto e médio prazo) }\end{array}$ \\
\hline $\begin{array}{l}\text { Assistência } \\
\text { laboratorial }\end{array}$ & $\begin{array}{l}\text { - Estrutura física } \\
\text { - Geladeira } \\
\text { - Termômetro } \\
\text { - Computador } \\
\text { - Kits para teste } \\
\text { rápido anti-HIV } \\
\text { - Kits para teste } \\
\text { rápido de sífilis }\end{array}$ & $\begin{array}{l}\text { - Armazenamento de } \\
\text { medicamento de forma } \\
\text { adequadae no prazo de validade } \\
\text { - Reposição de medicamentos } \\
\text { - Registro da distribuição de } \\
\text { medicamentos em livros ou em } \\
\text { sistemas informatizados } \\
\text { - Serviços em condições físicas } \\
\text { adequadas } \\
\text { - Equipamentos adequados } \\
\text { para realização dos exames } \\
\text { - Realização de exames para } \\
\text { diagnóstico da sífilis: teste } \\
\text { rápido e VDRL } \\
\text { - Estabelecimento de critério } \\
\text { de tempo para entrega do } \\
\text { teste rápido de HIV e sífilis } \\
\text { - Encaminhamento para } \\
\text { exames confirmatórios } \\
\text { - Estabelecimento de critério } \\
\text { de tempo para entrega de } \\
\text { exame confirmatório } \\
\text { - Realização de amostra cega } \\
\text { para controle de qualidade dos } \\
\text { testes rápidos para HIV e sífilis } \\
\text { - Monitoramento de entrada e } \\
\text { saída de exames informatizados }\end{array}$ & $\begin{array}{l}\text { - Garantia de realização do teste } \\
\text { rápido para triagem da infeç̧ão } \\
\text { pelo HIV } \\
\text { - Garantia de realização do teste } \\
\text { rápido e VDRL para identificação } \\
\text { da síilis gestacional e congênita } \\
\text { - Estabelecimento de tempo para } \\
\text { entrega de exames solicitados } \\
\text { - Mecanismo de controle de } \\
\text { qualidade para testagem através } \\
\text { de amostras cegas } \\
\text { - Adoção de critérios informatizados } \\
\text { de entrada e saída de exames }\end{array}$ & $\begin{array}{l}\text { - Parturientes atendidas com } \\
\text { os exames para triagem do } \\
\text { HIV e da sífilis } \\
\text { - Determinação do tempo de } \\
\text { envio e disponibilidade do } \\
\text { resultado dos testes rápido } \\
\text { e confirmatório } \\
\text { - Estabelecimento de } \\
\text { mecanismos para controle } \\
\text { da qualidade de testagem } \\
\text { dos exames de HIV e sífilis }\end{array}$ \\
\hline $\begin{array}{l}\text { Assistência } \\
\text { clínica }\end{array}$ & $\begin{array}{l}\text { - Estrutura física } \\
\text { - Geladeira } \\
\text { - Manuais e normas } \\
\text { técnicas } \\
\text { - Partograma }\end{array}$ & $\begin{array}{l}\text { - Estrutura física adequada na } \\
\text { triagem e pré-parto } \\
\text { - Equipamento médico-hospitalar } \\
\text { - Acompanhante no pré-parto } \\
\text { - Conhecimento sobre os } \\
\text { resultados dos exames de HIV } \\
\text { e síilis no pré-natal } \\
\text { - Coleta de sangue para } \\
\text { testagem de HIV e sífilis ao } \\
\text { chegar à maternidade } \\
\text { - Consentimento e aconselha- } \\
\text { mento sobre os exames coletados } \\
\text { - Exame obstétrico antes do parto } \\
\text { - Partograma preenchido } \\
\text { - Evolução do trabalho de parto } \\
\text { no prontuário } \\
\text { - Via de parto definida de acordo } \\
\text { com as recomendações } \\
\text { - Visitas médica e de enfermagem } \\
\text { no pós-parto } \\
\text { - Encaminhamento dos exames } \\
\text { positivos para teste confirmatório } \\
\text { - Administração do tratamento } \\
\text { anti-retroviral à gestante e } \\
\text { recém-nascido HIV+ } \\
\text { - Realização de inibição mecânica }\end{array}$ & $\begin{array}{l}\text { - Estrutura física adequada } \\
\text { - Disponibilidade de equipamento } \\
\text { médico-hospitalar em boas } \\
\text { condições de uso } \\
\text { - Permissão para acompanhamento } \\
\text { durante o internamento } \\
\text { - Utilização do partograma para } \\
\text { evolução e definição da via de parto } \\
\text { - Parturientes testadas para o } \\
\text { teste anti-HIV e sífilis } \\
\text { - Parturientes positivas em testes } \\
\text { rápidos testadas em exame } \\
\text { confirmatório } \\
\text { - Utilização de medidas } \\
\text { terapêuticas para gestantes e } \\
\text { recém-nascidos positivos para } \\
\text { o HIV e/ou sífilis } \\
\text { - Puérperas HIV+ orientadas para } \\
\text { o risco de aleitamento materno } \\
\text { - Puérperas HIV+ orientadas para } \\
\text { o uso da fórmula infantil em } \\
\text { seu recém-nascido } \\
\text { - Inclusão na rotina de raio X } \\
\text { de ossos longos e análise do } \\
\text { líquor para os recém-nascidos } \\
\text { com sífilis congênita }\end{array}$ & $\begin{array}{l}\text { - Garantia de assistência } \\
\text { adequada à parturiente HIV+, } \\
\text { sífilis e recém-nascido exposto } \\
\text { - Redução do risco da } \\
\text { transmissão vertical do HIV e } \\
\text { sífilis por meio da assistência } \\
\text { adequada }\end{array}$ \\
\hline
\end{tabular}

(continua) 


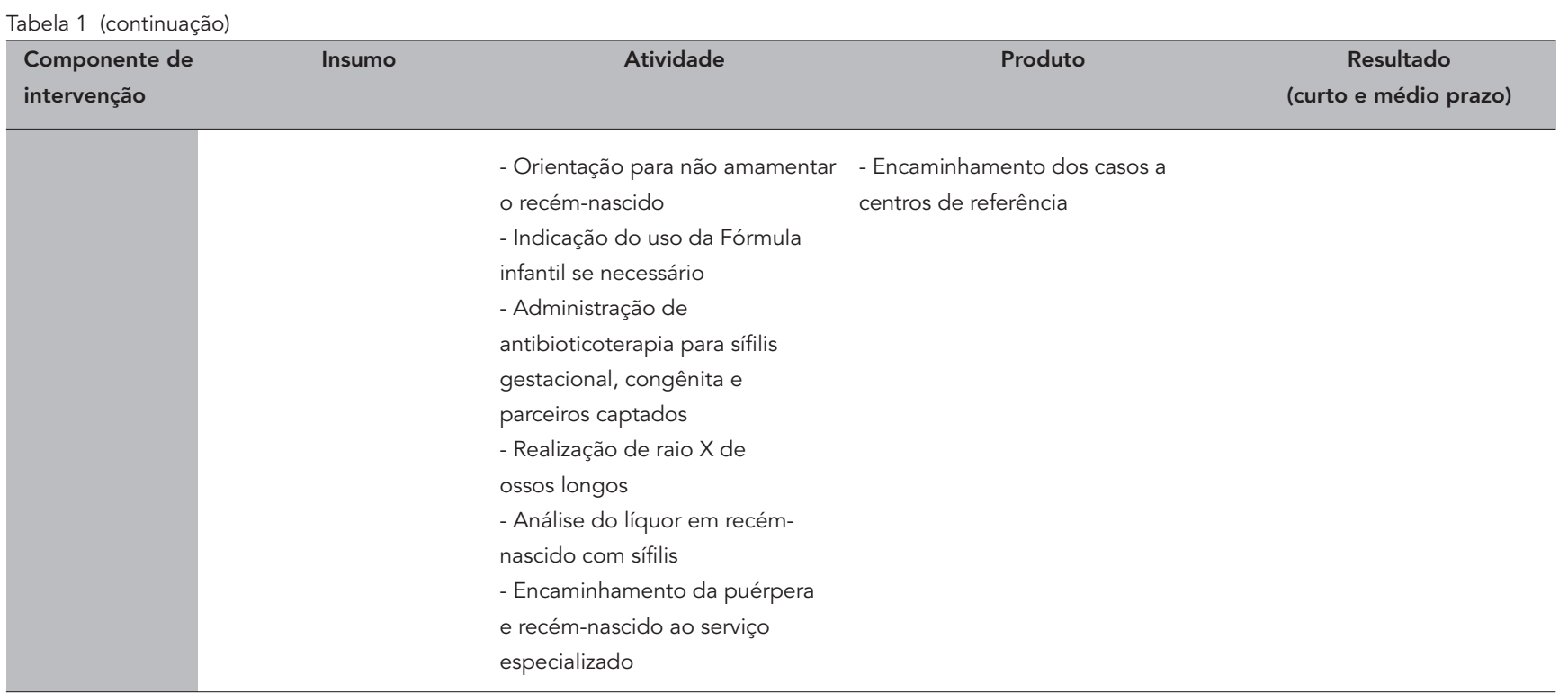

SIH: Sistema de Informações Hospitalares.

junho de 2005. Na Tabela 2 apresenta-se os indicadores selecionados para a avaliação segundo componentes e parte integrante do modelo que representam, além dos instrumentos de onde se obtiveram os dados.

A relação de prontuários a serem avaliados obedeceu a uma amostragem aleatória estratificada, amparada no número de nascimentos das maternidades e informados pelo Sistema de Informação sobre Nascidos Vivos (SINASC), disponibilizados pela Secretaria Estadual de Saúde de Pernambuco para o ano de 2004. O tamanho global da amostra foi calculado em um universo de 13.772 nascidos vivos dessas maternidades, fornecendo 430 prontuários para o interior do estado. Ao identificar a listagem dos prontuários para cada maternidade por ordem alfabética, selecionou-se de 5 em 5 até atingir o número necessário para cada serviço a ser avaliado. Para os casos de sífilis em gestantes, sífilis congênita e HIV positivo foi analisado o universo dos prontuários casos para o ano de 2004.

Para a realização da entrevista com as mães foram considerados $20 \%$ dos prontuários selecionados de forma sistemática, dentre as mulheres presentes na unidade, e obedecendo proporcionalmente os partos ocorridos no turno diurno ou noturno, alternando-se entre parto normal e cesáreo.

A observação da triagem e pré-parto correspondeu a $10 \%$ dos prontuários analisados, sendo o número não inferior a cinco observações por maternidade avaliada.

A definição adotada para o grau de implantação correspondeu à medida da integralidade das ações executadas para cada componente, sendo ordenados em quatro estratos: aceitável (90,1\% ou mais), parcialmente implantado $(50,1 \%$ a $90 \%)$, inaceitável (10,1\% a 50\%) e não implantado (até $10 \%$ ). O cálculo do escore do grau de implantação do Projeto Nascer foi obtido pelo somatório dos valores encontrados para cada um dos seus componentes.

O estudo atende a Resolução $n^{\circ}$. 196/96 do Conselho Nacional de Saúde e foi aprovado pelo Comitê de Ética em Pesquisa com Seres Humanos do Instituto de Medicina Integral Professor Fernando Figueira (IMIP).

\section{Resultados}

A Tabela 3 reúne as informações relacionadas ao grau de implantação do Projeto Nascer segundo componentes e a sua dimensão unitária. No que tange ao componente de prevenção verificou-se que todas as maternidades-casos apresentaram o mesmo desempenho, categorizado como inaceitável. A maternidade D apresentou um valor diferenciado (35\%), porém, na mesma classificação, devido, principalmente, ao melhor desempenho entre os serviços avaliados do indicador cartão do pré-natal da parturiente para maternidade.

A vigilância epidemiológica se configurou com baixa adesão, enquadrando-se na categoria inaceitável, com exceção da maternidade $\mathrm{A}$ $(68,75 \%)$ que atingiu a condição de parcialmente implantado. Esse serviço possui comissão de análise de óbito e controle de infecção hospi- 
Tabela 2

Indicadores, parte integrante do modelo, questionário onde se obtiveram os dados e padrão adotado para definição do grau de implantação dos componentes de prevenção, vigilância epidemiológica, assistência farmacêutica, laboratorial e clínica.

\begin{tabular}{|c|c|c|c|c|c|}
\hline $\begin{array}{l}\text { Componente } \\
\text { do modelo }\end{array}$ & Indicador & $\begin{array}{c}\text { Parte } \\
\text { integrante } \\
\text { do modelo }\end{array}$ & Questionário & Padrão & $\begin{array}{l}\text { Categoria e } \\
\text { ponto de corte }\end{array}$ \\
\hline \multirow[t]{5}{*}{ Prevenção } & $\begin{array}{c}\text { Cartão do pré-natal da parturiente } \\
\text { para maternidade }\end{array}$ & Insumo & Mulher & & $\begin{array}{c}\geq 10 \%=0,00 \\
10,1 \%-50 \%=0,50 \\
50,1 \%-90 \%=0,75 \\
90,1 \text { e mais }=1,0\end{array}$ \\
\hline & Autorização para realização do teste rápido & Insumo & $\begin{array}{c}\text { Observação, } \\
\text { triagem e } \\
\text { pré-parto }\end{array}$ & & $\begin{array}{c}\geq 10 \%=0,00 \\
10,1 \%-50 \%=0,50 \\
50,1 \%-90 \%=0,75 \\
90,1 \text { e mais }=1,0\end{array}$ \\
\hline & $\begin{array}{l}\text { Aconselhamento sobre a importância } \\
\text { do exame de sífilis }\end{array}$ & Atividade & $\begin{array}{c}\text { Observação, } \\
\text { triagem e } \\
\text { pré-parto }\end{array}$ & & $\begin{array}{c}\geq 10 \%=0,00 \\
10,1 \%-50 \%=0,50 \\
50,1 \%-90 \%=0,75 \\
90,1 \text { e mais }=1,0\end{array}$ \\
\hline & $\begin{array}{l}\text { Aconselhamento sobre a importância } \\
\text { do exame de HIV }\end{array}$ & Atividade & $\begin{array}{c}\text { Observação, } \\
\text { triagem e } \\
\text { pré-parto }\end{array}$ & & $\begin{array}{c}\geq 10 \%=0,00 \\
10,1 \%-50 \%=0,50 \\
50,1 \%-90 \%=0,75 \\
90,1 \text { e mais }=1,0\end{array}$ \\
\hline & $\begin{array}{l}\text { Normas afixadas para prevenção da } \\
\text { transmissão vertical do HIV e sífilis }\end{array}$ & Atividade & $\begin{array}{c}\text { Observação, } \\
\text { triagem e } \\
\text { pré-parto }\end{array}$ & & $\begin{array}{c}\geq 10 \%=0,00 \\
10,1 \%-50 \%=0,50 \\
50,1 \%-90 \%=0,75 \\
90,1 \text { e mais }=1,0\end{array}$ \\
\hline \multirow[t]{8}{*}{$\begin{array}{l}\text { Vigilância } \\
\text { epidemiológica }\end{array}$} & Comissão de Análise de Óbitos & Insumo & Institucional & Pelo menos 1 por maternidade & $\begin{array}{c}1(\operatorname{Sim})=100 \% \\
0 \text { (Não) }=0 \%\end{array}$ \\
\hline & Comissão de Revisão de Prontuário & Insumo & Institucional & Pelo menos 1 por maternidade & $\begin{array}{c}1(\operatorname{Sim})=100 \% \\
0 \text { (Não) }=0 \%\end{array}$ \\
\hline & Comissão de Controle e Infecção Hospitalar & Insumo & Institucional & Pelo menos 1 por maternidade & $\begin{array}{c}1(\operatorname{Sim})=100 \% \\
0(\text { Não })=0 \%\end{array}$ \\
\hline & Notificação de gestantes HIV em 2004 & Atividade & Institucional & $\begin{array}{l}\text { Pelo menos } 1 \text { caso notificado } \\
\text { de HIV em } 2004\end{array}$ & $\begin{array}{c}\geq 1 \text { caso }=100 \% \\
\text { Ausência de casos } \\
=0 \%\end{array}$ \\
\hline & Notificação de sífilis congênita em 2004 & Atividade & Institucional & $\begin{array}{l}\text { Pelo menos } 5 \text { casos notificados } \\
\text { de sífilis congênita em } 2004\end{array}$ & $\begin{array}{c}\geq 5 \text { casos }=100 \% \\
\text { Ausência de casos } \\
=0 \%\end{array}$ \\
\hline & Notificação de gestantes com sífilis em 2004 & Atividade & Institucional & $\begin{array}{l}\text { Pelo menos } 5 \text { casos notificados } \\
\text { de sífilis em gestantes em } 2004\end{array}$ & $\begin{array}{c}\geq 5 \text { casos }=100 \% \\
\text { Ausência de casos } \\
=0 \%\end{array}$ \\
\hline & Rastreamento de parceiro com sífilis & Atividade & Prontuário & $\begin{array}{l}\text { Testar todo parceiro de } \\
\text { gestante positiva para sífilis }\end{array}$ & $\begin{array}{c}\geq 10 \%=0,00 \\
10,1 \%-50 \%=0,50 \\
50,1 \%-90 \%=0,75 \\
90,1 \text { e mais }=1,0\end{array}$ \\
\hline & $\begin{array}{l}\text { Aconselhamento sobre o porquê da } \\
\text { realização dos exames de HIV e sífilis }\end{array}$ & Atividade & Mulher & & $\begin{array}{c}\geq 10 \%=0,00 \\
10,1 \%-50 \%=0,50 \\
50,1 \%-90 \%=0,75 \\
90,1 \text { e mais }=1,0\end{array}$ \\
\hline
\end{tabular}

(continua) 
Tabela 2 (continuação)

\begin{tabular}{lc}
\hline $\begin{array}{l}\text { Componente } \\
\text { do modelo }\end{array}$ & Indicador \\
\hline $\begin{array}{l}\text { Assistência } \\
\text { farmacêutica }\end{array}$ & Existência de telas em janela \\
Existência de geladeira \\
Medicamentos anti-retrovirais \\
(AZT: xarope, comprimido e injetáve \\
inibidor de lactação e fórmula láctea)
\end{tabular}

Parte

integrante

do modelo

Insumo

\section{Insumo}

Insumo

(AZT: xarope, comprimido e injetável,

$$
\text { inibidor de lactação e fórmula láctea) }
$$

Medicamento para tratamento da sífilis (penicilina benzatina, procaína e cristalina)

Serviço em condições físicas adequadas (presença de mofo, infiltração, luz solar, caixa de remédio no chão ou teto e parede)

A geladeira acondiciona alimentos e outros produtos

Registro de temperatura da geladeira

Controle do estoque

Assistência

laboratorial

Existência de geladeira

Existência de termômetro de geladeira

Kit teste rápido anti-HIV

Kit teste rápido sífilis

Realiza teste de identificação para HIV

Realiza exame VDRL

Atividade

Atividade

Atividade

Insumo

Insumo

Atividade

Atividade
Questionário

Categoria e

ponto de corte

Farmácia

Possuir em todas as janelas

$1(\operatorname{Sim})=100 \%$

0 (Não) $=0 \%$

Farmácia

Pelo menos 1 por farmácia

$1(\operatorname{Sim})=100 \%$

0 (Não) $=0 \%$

5 respostas

negativas $=0$

1 resposta

positiva $=0,5$

2 ou 3 respostas

positivas $=0,75$

4 respostas

positivas $=1$

Insumo Farmácia

3 respostas

negativas $=0$

1 resposta

positiva $=0,5$

2 respostas

positivas $=0,75$

3 respostas

positivas $=1$

Insumo Farmácia

3 respostas

positivas $=0$

1 resposta

negativa $=0,5$

2 respostas

negativas $=0,75$

3 respostas

negativas $=1$

Farmácia

Acondicionar exclusivamente

$1(\mathrm{Sim})=100 \%$

medicamentos

Farmácia

Possuir registro em toda geladeira

0 (Não) $=0 \%$

$1(\mathrm{Sim})=100 \%$

0 (Não) $=0 \%$

Farmácia

Realizar em toda farmácia

$1(\operatorname{Sim})=100 \%$

0 (Não) $=0 \%$

Insumo Laboratório

Pelo menos 1 por laboratório

$1(\operatorname{Sim})=100 \%$

0 (Não) $=0 \%$

Laboratório

Pelo menos 1 por geladeira

$1(\mathrm{Sim})=100 \%$

0 (Não) $=0 \%$

Laboratório

Possuir pelo menos 1

$1(\mathrm{Sim})=100 \%$

0 (Não) $=0 \%$

Insumo Laboratório

Possuir pelo menos 1

$1(\mathrm{Sim})=100 \%$

0 (Não) $=0 \%$

Laboratório

Realizar em todo serviço

$1(\mathrm{Sim})=100 \%$

0 (Não) $=0 \%$

Realizar em todo serviço

$1(\operatorname{Sim})=100 \%$

0 (Não) $=0 \%$

(continua) 
Tabela 2 (continuação)

\begin{tabular}{|c|c|c|c|c|c|}
\hline $\begin{array}{l}\text { Componente } \\
\text { do modelo }\end{array}$ & Indicador & $\begin{array}{c}\text { Parte } \\
\text { integrante } \\
\text { do modelo }\end{array}$ & Questionário & Padrão & $\begin{array}{c}\text { Categoria e } \\
\text { ponto de corte }\end{array}$ \\
\hline & $\begin{array}{l}\text { Referência para realizar exames confirmatórios } \\
\text { para HIV }\end{array}$ & Atividade & Laboratório & Realizar em todo serviço & $\begin{array}{c}1(\operatorname{Sim})=100 \% \\
0(\text { Não })=0 \%\end{array}$ \\
\hline & Tempo de entrega do testes rápidos de HIV & Atividade & Laboratório & Até 30 minutos & $\begin{array}{l}\geq 30 \text { minutos }=100 \% \\
31-40 \text { minutos }=75 \% \\
41 \text { minutos }- \\
1 \text { hora }=50 \%\end{array}$ \\
\hline & $\begin{array}{l}\text { Tempo de entrega do testes confirmatórios } \\
\text { de sífilis }\end{array}$ & Atividade & Laboratório & Até 24 horas & $\begin{array}{l}\leq 24 \text { horas } \\
\text { (adequado) }=100 \% \\
\quad \geq 24 \text { horas } \\
\text { (inadequado) }=0,0 \%\end{array}$ \\
\hline & $\begin{array}{l}\text { Referência para realizar exames } \\
\text { confirmatórios de sífilis }\end{array}$ & Atividade & Laboratório & $\begin{array}{c}\text { Realizar referência em todos } \\
\text { os casos positivos }\end{array}$ & $\begin{array}{c}1(\operatorname{Sim})=100 \% \\
0(\text { Não })=0 \%\end{array}$ \\
\hline & Controle de qualidade do VDRL & Atividade & Laboratório & $\begin{array}{l}\text { Realizar } 1 \text { a cada novo } \\
\text { lote dos kits }\end{array}$ & $\begin{array}{c}1(\operatorname{Sim})=100 \% \\
0(\text { Não })=0 \%\end{array}$ \\
\hline \multirow[t]{9}{*}{$\begin{array}{l}\text { Assistência } \\
\text { clínica }\end{array}$} & $\begin{array}{l}\text { Equipamento para auscultar os batimentos } \\
\text { cardio-fetais }\end{array}$ & Insumo & $\begin{array}{l}\text { Observação, } \\
\text { triagem e } \\
\text { pré-parto }\end{array}$ & Pelo 1 menos por triagem & \\
\hline & $\begin{array}{l}\text { Serviço com condições físicas adequadas } \\
\text { (temperatura ambiente, cama forrada, } \\
\text { ausência de lixo e de restos de alimentos) }\end{array}$ & Insumo & $\begin{array}{l}\text { Observação, } \\
\text { triagem e } \\
\text { pré-parto }\end{array}$ & & $\begin{array}{c}\text { Nenhum adequado }=0 \\
1 \text { item adequado }=0,5 \\
2 \text { ou } 3 \text { itens } \\
\text { adequados }=0,75 \\
\text { Todos os itens } \\
\text { adequados }=1\end{array}$ \\
\hline & Presença do partograma no prontuário & Insumo & Prontuário & & $\begin{array}{c}\geq 10 \%=0,00 \\
10,1 \%-50 \%=0,50 \\
50,1 \%-90 \%=0,75 \\
90,1 \text { e mais }=1,0\end{array}$ \\
\hline & Permissão de acompanhamento & Atividade & Institucional & Permitir a toda parturiente & \\
\hline & Realização do teste rápido para HIV na triagem & Atividade & $\begin{array}{l}\text { Observação, } \\
\text { triagem e } \\
\text { pré-parto }\end{array}$ & & $\begin{array}{c}\geq 10 \%=0,00 \\
10,1 \%-50 \%=0,50 \\
50,1 \%-90 \%=0,75 \\
90,1 \text { e mais }=1,0\end{array}$ \\
\hline & $\begin{array}{c}\text { Informação sobre a realização desses } \\
\text { dois exames (HIV e sífilis) }\end{array}$ & Atividade & Mulher & & $\begin{array}{c}\geq 10 \%=0,00 \\
10,1 \%-50 \%=0,50 \\
50,1 \%-90 \%=0,75 \\
90,1 \text { e mais }=1,0\end{array}$ \\
\hline & Examinada antes do parto & Atividade & Mulher & & $\begin{array}{c}\geq 10 \%=0,00 \\
10,1 \%-50 \%=0,50 \\
50,1 \%-90 \%=0,75 \\
90,1 \text { e mais }=1,0\end{array}$ \\
\hline & Número de visitas médicas no pós-parto & Atividade & Prontuário & & $\begin{array}{c}\geq 10 \%=0,00 \\
10,1 \%-50 \%=0,50 \\
50,1 \%-90 \%=0,75 \\
90,1 \text { e mais }=1,0\end{array}$ \\
\hline & $\begin{array}{l}\text { Registro no prontuário sobre o } \\
\text { risco do HIV e sífilis }\end{array}$ & Atividade & Prontuário & & $\begin{array}{c}\geq 10 \%=0,00 \\
10,1 \%-50 \%=0,50 \\
50,1 \%-90 \%=0,75 \\
90,1 \text { e mais }=1,0\end{array}$ \\
\hline
\end{tabular}

(continua) 


\begin{tabular}{|c|c|c|c|c|c|}
\hline \multirow[t]{13}{*}{ do modelo } & Indicador & $\begin{array}{c}\text { Parte } \\
\text { integrante } \\
\text { do modelo }\end{array}$ & Questionário & Padrão & $\begin{array}{l}\text { Categoria e } \\
\text { ponto de corte }\end{array}$ \\
\hline & Raio $X$ de ossos longos nos casos positivos & Atividade & Prontuário & & $\geq 10 \%=0,00$ \\
\hline & & & & & $10,1 \%-50 \%=0,50$ \\
\hline & & & & & $50,1 \%-90 \%=0,75$ \\
\hline & & & & & 90,1 e mais $=1,0$ \\
\hline & Análise do líquor em recém-nascido com sífilis & Atividade & Prontuário & & $\geq 10 \%=0,00$ \\
\hline & & & & & $10,1 \%-50 \%=0,50$ \\
\hline & & & & & $50,1 \%-90 \%=0,75$ \\
\hline & & & & & 90,1 e mais $=1,0$ \\
\hline & Encaminhar puérpera com sífilis & Atividade & Prontuário & & $\geq 10 \%=0,00$ \\
\hline & para centro especializado & & & & $10,1 \%-50 \%=0,50$ \\
\hline & & & & & $50,1 \%-90 \%=0,75$ \\
\hline & & & & & 90,1 e mais $=1,0$ \\
\hline
\end{tabular}

Tabela 3

Grau de implantação do Projeto Nascer segundo componentes e dimensão unitária em quatro maternidades do interior de Pernambuco, Brasil, 2005.

\begin{tabular}{|c|c|c|c|c|c|c|c|c|c|}
\hline \multirow[t]{2}{*}{ Componentes } & \multirow{2}{*}{$\begin{array}{l}\text { Pontuação } \\
\text { máxima }\end{array}$} & \multicolumn{2}{|c|}{ Maternidade A } & \multicolumn{2}{|c|}{ Maternidade B } & \multicolumn{2}{|c|}{ Maternidade C } & \multicolumn{2}{|c|}{ Maternidade D } \\
\hline & & $\mathrm{n}$ & $\%$ & $\mathrm{n}$ & $\%$ & $\mathrm{n}$ & $\%$ & $\mathbf{n}$ & $\%$ \\
\hline Prevenção & 5 & 0,75 & 15,00 & 0,75 & 15,00 & 0,75 & 15,00 & 1,75 & 35,00 \\
\hline Vigilância epidemiológica & 8 & 5,5 & 68,75 & 4 & 50,00 & 1 & 12,50 & 3,5 & 43,75 \\
\hline Assistência farmacêutica & 8 & 4,5 & 56,25 & 7 & 87,50 & 5,25 & 65,63 & $5, .25$ & 65,63 \\
\hline Assistência laboratorial & 11 & 9,75 & 88,64 & 8 & 72,73 & 2 & 18,18 & 7 & 63,64 \\
\hline Assistência clínica & 12 & 7 & 58,33 & 6 & 50,00 & 4,5 & 37,50 & 3,75 & 31,25 \\
\hline Dimensão unitária & 44 & 27,5 & 62,50 & 25,75 & 58,52 & 13,5 & 30,68 & 21,25 & 48,30 \\
\hline
\end{tabular}

Legenda:

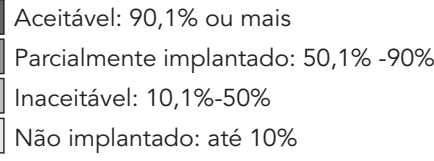

talar, além de apresentar casos notificados de gestantes com HIV, sífilis e recém-nascido com sífilis congênita. O não enquadramento na condição de "aceitável" decorreu da ausência de comissão de revisão de prontuário e rastreamento do parceiro com sífilis.

Para os componentes de assistência farmacêutica e laboratorial, grande parte dos serviços foi classificada como parcialmente implantado, exceto, a maternidade C $(18,18 \%)$ que neste último componente obteve a condição de inaceitável. A maternidade $C$ não possuía o kit teste rápido anti-HIV e referência para realizar exames confirmatórios para HIV e sífilis. Em todas as maternidades avaliadas foi constatada ausência de pelo menos um medicamento padronizado pelo Ministério da Saúde para o tratamento da transmissão vertical do HIV.

Em relação à estrutura organizacional da farmácia avaliou-se a adequação do armazenamento dos medicamentos. A análise revelou que em metade das farmácias existiam medicamentos expostos à luz solar e caixas em contato com o chão e a parede. Quanto ao componente assistência laboratorial, chama a atenção a ausência de teste rápido para sífilis no universo das maternidades e o tempo de entrega do teste rápido para HIV que ultrapassa o recomendado em metade dos serviços avaliados. 
Na assistência clínica, devido à fragilidade de ações assistenciais para redução da transmissão vertical do HIV e sífilis, a situação avaliada revela que as maternidades B (50\%), C $(37,5 \%)$ e D $(31,25 \%)$ foram categorizadas como inaceitável e a maternidade A $(58,33 \%)$ como parcialmente implantado. Os indicadores realização do teste rápido para HIV na triagem e a informação sobre a realização desses dois exames (HIV e sífilis) apresentaram baixa adesão em todas as maternidades.

Nesse componente também foram investigadas as informações contidas nos prontuários de gestantes e crianças expostas ao HIV, como o uso do inibidor de lactação, AZT injetável, xarope e fórmula infantil segundo questionário prontuário. $\mathrm{O}$ resultado mostrou a ausência de registros médicos e de outros profissionais de saúde nos prontuários, o que dificultou avaliar e aprofundar a qualidade da assistência oferecida.

Em relação ao grau de implantação da dimensão unitária do Projeto Nascer, representado por uma síntese de cada um dos cinco componentes, considerando os critérios adotados (Tabela 3), o melhor desempenho foi o da maternidade A $(62,5 \%)$, seguida da maternidade B $(58,52 \%)$, classificadas como parcialmente implantado. As maternidades C $(30,68 \%)$ e D $(48,3 \%)$ foram consideradas na categoria inaceitável.

\section{Discussão}

A análise apresentada confirma que o Projeto Nascer não está implantado nas maternidades avaliadas. A utilização do modelo lógico e seus respectivos indicadores foram importantes para verificar quais atividades normatizadas estão sendo realizadas nos serviços e estão contribuindo para redução da transmissão vertical das infecções. A abordagem metodológica utilizada valorizou múltiplas técnicas de coletas e a diversidade de informantes para garantir maior robustez ao estudo. No entanto, o foco preferencial do estudo foi a análise do grau de implantação, sendo valorizada a síntese dos aspectos normatizados por componente e a dimensão unitária do Projeto Nascer. Quanto aos indicadores selecionados, entre todos os componentes, mesmo os que são considerados como fundamentais para implantação do projeto, existiram ausências de informações em diversas fontes de pesquisa. Entretanto, o indicador não foi retirado devido à sua importância.

O componente prevenção encontra-se no modelo lógico, embora o Projeto Nascer tenha sido construído para ser implementado em maternidades, no segundo nível de atenção. Todavia, o Programa Nacional de DST/AIDS recomenda incluir práticas de prevenção nas atividades assistenciais já existentes nos serviços de saúde 19 . Um indicador fundamental ao componente prevenção foi o aconselhamento, considerado a ação desencadeadora de todas as ações de prevenção da transmissão vertical, quando as mulheres teriam o conhecimento dos benefícios da profilaxia para o seu bebê, como também para si mesma 21

Os resultados mostraram ausência das ações de aconselhamento e autorização para realização do teste rápido nas maternidades-casos. Pode-se constatar que os profissionais de saúde, ao atenderem a parturiente, na observação triagem e pré-parto, solicitavam os exames como rotina ou conduta instituída pelo serviço, não registrando nos prontuários a autorização e a prática de aconselhamento para realização dos exames. A inexistência de aconselhamento é uma situação comum em outros serviços de saúde. Estudo em diferentes estabelecimentos de saúde - que incluía centros de saúde, maternidades e unidades de saúde da família - comprovou que nenhum serviço promoveu aconselhamento, mesmo quando solicitou o teste anti-HIV 22.

Santos et al. 23 já chamavam a atenção para os baixos percentuais de aconselhamento em um centro de referência. Apenas um terço das mulheres declarou receber algum apoio no aconselhamento pós-teste; a maioria não recebeu nenhum aconselhamento ou foi simplesmente encaminhada ao serviço de referência.

Para orientar e subsidiar as ações de aconselhamento faz-se necessário incorporar ações de vigilância epidemiológica tornando disponíveis informações relevantes, confiáveis e atualizadas. Nesse sentido, os indicadores que fazem parte do componente de vigilância epidemiológica permitirão avaliar a comissão de análise de óbitos, revisão de prontuário, controle de infecção hospitalar, notificação de sífilis congênita, notificação de gestantes com HIV e sífilis, rastreamento de parceiros com sífilis e o aconselhamento sobre o porquê da realização dos exames de HIV e sífilis.

Em relação à existência da comissão de análise de óbitos, revisão de prontuário e comissão de controle de infecção hospitalar, apenas esta última foi verificada em todas as maternidadescasos. Observou-se também que outras comissões não foram implementadas para investigar os casos positivos de HIV e sífilis, condição que poderia levar a mudanças de atitudes dos profissionais de saúde 24

Outro aspecto avaliado foi o rastreamento do parceiro com sífilis, o que permitiu verificar o quanto é baixo o percentual de captação pa- 
ra o tratamento concomitante. Esse indicador demonstrou a fragilidade do serviço no que se refere a uma assistência de qualidade, bem como o risco de um tratamento ineficaz. Durante o período da pesquisa, percebeu-se que as gestantes e as puérperas, mesmo de alta hospitalar, não recebiam orientação para o tratamento de seu companheiro, embora o Ministério da Saúde 19 oriente sobre a necessidade do parceiro fazer o exame de VDRL, com a finalidade de realizar o tratamento.

Basso et al. 25 , ao analisarem a prevalência da sífilis congênita ocorrida em um hospital de Caxias do Sul (Rio Grande do Sul), constataram que em $53,2 \%$ dos casos o parceiro não foi tratado simultaneamente com a gestante. O estudo de Schetini et al. 26 , ao descrever a prevalência da sífilis congênita em um hospital público de Niterói (Rio de Janeiro), revelou percentuais de 1,92\% a $15,38 \%$ para os parceiros que foram tratados.

Com o propósito de superar os baixos desempenhos, o serviço de saúde deve oferecer o aconselhamento, testagem rápida e tratamento aos parceiros captados e para as mulheres. Considerando esse fato, ações dos componentes de assistência farmacêutica e laboratorial, tais como o acesso à terapêutica medicamentosa e aos testes rápidos, são fundamentais para reorganização e eficiência do programa 27.

Em relação ao componente de assistência farmacêutica, destaca-se a disponibilidade dos medicamentos anti-retrovirais (ARV). Verificouse a ausência do AZT xarope apenas em uma maternidade. Segundo a política de distribuição de medicamentos, cabe à esfera federal a compra dos ARV e às esferas municipal e estadual a compra dos demais. O compromisso de manter o acesso universal à terapia ARV tem diferenciado positivamente a luta contra a AIDS no Brasil 27.

Vasconcelos 28, ao avaliar a assistência prestada a parturientes HIV positivas e seus recémnascidos em quatro capitais brasileiras, revelou que $90,1 \%$ dos bebês utilizaram a profilaxia com AZT solução oral. O fato de um dos serviços não disponibilizar a medicação indica uma fragilidade da assistência, uma vez que o tempo para início da terapia se constitui em fator de proteção essencial a não soropositividade para o HIV.

Outro ponto relevante foi a ausência do AZT comprimido em três maternidades, e mesmo sendo mais utilizado durante o período do pré-natal, não se pode deixar de enfatizar que as farmácias avaliadas são referência para o atendimento às gestantes. Cavalcante et al. 21, ao analisarem as condutas de redução da transmissão vertical do HIV nas grávidas infectadas, nas maternidades públicas de Fortaleza (Ceará), verificaram que das 109 mulheres que tiveram atendimento de pré-natal, 101 poderiam ter sido submetidas a todas as etapas da profilaxia para a redução da transmissão vertical porque conheciam seu status sorológico antes ou durante a gravidez, entretanto, somente 48 receberam a profilaxia completa.

Vasconcelos 28 também identificou, pela análise dos prontuários, o uso da terapia anti-retroviral (TARV) pelas gestantes, parturientes e seus recém-nascidos. Os resultados mostraram que 359 mulheres (24\%) não usaram o AZT durante a sua gestação; 282 parturientes (19\%) não foram medicadas com o AZT intravenoso; e em 112 bebês (8\%) não foi administrado o AZT solução oral, mesmo em maternidades de referência.

Estudo realizado com o objetivo de determinar a prevalência de testagem anti-HIV em parturientes admitidas no IMIP, apontou que das 154 mulheres que chegaram à triagem sem o resultado do teste anti-HIV do pré-natal, 134, ou seja, para $87 \%$ foi solicitado o teste rápido, no entanto, apenas $84,5 \%$ realizaram o exame nesse momento. Quanto ao recebimento do resultado, observou-se que 125 mulheres (96,2\%) afirmaram que não o receberam 29.

Em trabalho realizado numa maternidade pública de Teresina (Piauí), em 2003, para avaliar as intervenções realizadas para a prevenção da transmissão vertical do HIV, constatou contradições importantes entre os depoimentos dos profissionais de saúde e o número de exames ${ }^{1}$. A média mensal foi de 1.235 consultas de pré-natal em gestantes no primeiro trimestre, a média de exames anti-HIV foi de 400 ao mês, indicando uma cobertura de apenas $32,4 \%$.

Os testes rápidos para sífilis ainda não são bem aceitos pelos profissionais de saúde, mesmo com a ampliação dos serviços de atenção primária, incluindo os laboratórios. A utilização do teste em maior escala possibilitaria iniciar o tratamento desde o primeiro contato, diminuindo os casos de tratamento tardio ou não tratamento 30 .

Quanto ao componente assistência clínica, buscou-se incluir os indicadores existentes entre o normatizado e o concretizado no cotidiano das práticas e serviços de saúde. Esse componente é imprescindível para a diminuição da transmissão vertical das infecções abordadas, entretanto, os resultados apontaram um distanciamento existente entre as normas e medidas recomendadas e a realidade encontrada. Verificou-se que apenas uma maternidade foi caracterizada como parcialmente implantada.

É importante enfatizar que o modelo assistencial proposto pelo SUS ainda não é uma realidade nas maternidades avaliadas, e que o cumprimento de normas e diretrizes do Proje- 
to Nascer no momento do parto pode diminuir consideravelmente as chances de transmissão da infecção 16. Estudos avaliativos realizados em outros municípios do Brasil também apontam a fragilidade da assistência em diferentes aspectos $1,6,11,12,13,14,20$.

Foi observado que a realização do teste rápido para HIV na triagem apresentou pouca efetividade entre o universo das maternidades. Esse local é a porta de entrada da maternidade, significando que, se o teste for realizado nesse momento, tanto mais rápido será o diagnóstico e suas possíveis intervenções 31 . Souza Júnior et al. 3 apontam que falhas e os estrangulamentos do sistema de saúde dificultam a detecção da infecção pelo HIV entre as gestantes em todas as etapas do processo, desde a inclusão da gestante para o acompanhamento pré-natal até a ausência de pedido do teste sorológico para HIV e falta de conhecimento do resultado do teste antes do parto.

Em relação à assistência aos recém-nascidos portadores de sífilis congênita, verificou-se que apenas duas maternidades disponibilizaram o exame de raio $\mathrm{X}$ de ossos longos e análise do líquor, enquanto os protocolos recomendam a realização destes exames em todo caso positivo de sífilis congênita 20 . Estudo retrospectivo 32 sobre a prevalência de sífilis congênita realizado em Carapicuíba (São Paulo), em 2002, encontrou

\section{Resumo}

Este artigo avalia a assistência às mulheres em quatro maternidades públicas do Nordeste brasileiro quanto às medidas de intervenção adotadas pelo Projeto Nascer para redução da transmissão vertical do HIV e sífilis, em 2005. Trata-se de um estudo de casos múltiplos, abrangendo uma abordagem retrospectiva e outra transversal. O modelo lógico da intervenção tem cinco componentes: prevenção, vigilância epidemiológica, assistência farmacêutica, laboratorial e clínica. Para cada componente selecionaram-se indicadores de estrutura e processo. As maternidades foram classificadas em: aceitável, parcialmente implantado, inaceitável e não implantado. A maior aderência às normas foi observada na maternidade A (62,5\%), classificada em parcialmente implantada, e a menor na maternidade C (30,6\%), enquadrada como inaceitável. O estudo destaca a importância das ações de prevenção, vigilância epidemiológica, assistência farmacêutica, laboratorial e clínica para redução da transmissão vertical do HIV e da sífilis.

Avaliação em Saúde; Transmissão Vertical de Doenças; HIV; Sífilis na amostragem de 6.719 nascimentos, 32 casos de sífilis congênita, e destes, $100 \%$ foram submetidos ao diagnóstico radiológico e $97 \%$ a punção lombar.

O encaminhamento da puérpera com sífilis ao centro especializado foi realizado, apenas, por uma maternidade, e ainda assim, com baixos percentuais. $\mathrm{O}$ referido achado reforça a idéia de que a responsabilidade sanitária precisa ser implementada, garantindo o acompanhamento em serviços especializados até a negativação sorológica do VDRL. É verdadeira a ponderação de Schetini et al. 26 ao considerar a sífilis congênita uma urgência médica, pois quanto mais precocemente for tratada, maior a chance de se eliminar a infecção e as seqüelas no recém-nascido.

Este estudo pretendeu contribuir para o aprimoramento das ações que buscam diminuir a transmissão materno-infantil do HIV e da sífilis em quatro maternidades do interior de Pernambuco. Apesar dos desiguais graus de implantação, com amplitude de adesão e normas variando de $30,7 \%$ a $62,5 \%$, ele aponta para a necessidade de ações articuladas envolvendo gestores do sistema de saúde, gerentes das unidades e profissionais de saúde na busca da superação dos problemas identificados para possibilitar a reversão no país da situação desfavorável da transmissão vertical das infecções abordadas.

\section{Colaboradores}

V. C. Macêdo, A. F. B. Bezerra e P. G. Frias participaram da concepção, elaboração e redação do artigo. C. L. T. Andrade participou da construção da amostra e revisão do artigo.

\section{Agradecimentos}

À Secretaria de Saúde de Pernambuco pela disponibilização dos dados da pesquisa, a Ivanise Tibúrcio Cavalcanti e aos residentes do IMIP pelo trabalho de campo, e a Maria Goretti Godoy Sousa que elaborou a macro proposta da avaliação, participou da coleta, construiu e partilhou o banco de dados referente às maternidades avaliadas no interior de Pernambuco. 


\section{Referências}

1. Araújo LM, Nogueira LT. Transmissão vertical do HIV: situação encontrada em uma maternidade de Teresina. Rev Bras Enferm 2007; 60:396-9.

2. Programa Nacional de DST/AIDS, Ministério da Saúde. Diretrizes para o controle da sífilis congênita. Brasília: Ministério da Saúde; 2005.

3. Souza Júnior PRB, Szwarcwald CL, Barbosa Júnior A, Carvalho MFC, Castilho EA. Infecção pelo HIV durante a gestação: estudo - sentinela parturiente, Brasil, 2002. Rev Saúde Pública 2004; 38:764-72.

4. Nogueira SA, Abreu T, Oliveira R, Araújo L, Costa T, Andrade M, et al. Successful prevention of HIV transmission from mother to infant in Brazil using a multidisciplinary team approach. Braz J Infect Dis 2001; 5:78-86.

5. Succi RCM; Grupo de Estudo da Sociedade Brasileira de Pediatria para Avaliar a Transmissão Materno-Infantil do HIV. Mother-to-child transmission of HIV in Brazil during the years 2000 and 2001: results of a multi-centric study. Cad Saúde Pública 2007; 23 Suppl 3:S379-89.

6. Santos EM, Westman S, Reis AM, Alves RG. Avaliação do grau de implementação do programa de controle de transmissão vertical do HIV em maternidades do "Projeto Nascer". http://www. aids.gov.br/services/DocumentManagement/ FileDownload.EZTSvc.asp?DocumentID=\%7BE4 3AD660-0492-42CA-80DF-FF06610A1BD6\%7D\&S erviceInstUID=\%7BB8EF5DAF-23AE-4891-AD361903553A3174\%7D (acessado em 20/Mar/2009).

7. Saraceni V, Guimarães MHFS, Theme Filha MM, Leal MM. Mortalidade perinatal por sífilis congênita: indicador da qualidade da atenção à mulher e à criança. Cad Saúde Pública 2005; 21:1244-50.

8. Madi JM, De Lorenzi DRS. Sífilis congênita como indicador de assistência pré-natal. Rev Bras Ginecol Obstet 2001; 23:647-52.

9. Paz LC, Pereira FG, Matida LH, Saraceni V, Ramos Jr. AN. Vigilância epidemiológica da sífilis congênita no Brasil: definição de casos. Boletim Epidemiológico AIDS/DST 2004; Ano I, nº 1.

10. Carvalho FT, Piccinini CA. Aspectos históricos do feminino e do maternal e a infecção pelo HIV em mulheres. Ciênc Saúde Coletiva 2008; 13:1889-98.

11. Szwarcwald CL, Bastos FI, Esteves MAP, Andrade CLT. A disseminação da epidemia da AIDS no Brasil, no período de 1987-1996: uma análise espacial. Cad Saúde Pública 2000; 16 Suppl 1:7-19.

12. Tomazelli J, Czeresnia D, Barcellos C. Distribuição dos casos de AIDS em mulheres no Rio de Janeiro, de 1982 a 1997: uma análise espacial. Cad Saúde Pública 2003; 19:1049-61.

13. Dal Fabbro AL, Gurgel RQ, Lemos LMD. Prevalência da infecção por HIV em parturientes de maternidades vinculadas ao SUS. Rev Bras Ginecol Obstet 2005; 27:32-6.

14. Farias JPQ, Franco AS, Kleber P, Dourado I, Castro BG. Prevenção da transmissão vertical do HIV: atitude dos obstetras em Salvador, Brasil. Rev Bras Ginecol Obstet 2008; 30:135-41.

15. Ministério da Saúde. Portaria Ministerial nº 2.104 . Institui, no âmbito do Sistema Único de Saúde/ SUS, o Projeto Nascer. Diário Oficial da União 2002; 19 nov.
16. Programa Nacional de DST e AIDS, Ministério da Saúde. Projeto Nascer. Brasília: Programa Nacional de DST e AIDS; 2003.

17. Secretaria Estadual de Saúde de Pernambuco/Instituto Materno Infantil de Pernambuco. Relatório de construção da amostra da pesquisa avaliativa do Projeto Nascer - maternidades. Recife: Secretaria Estadual de Saúde de Pernambuco/Instituto Materno Infantil de Pernambuco; 2005.

18. Yin RK. Estudo de caso: planejamento e métodos. 3a Ed. Porto Alegre: Editora Artmed Bookman: 2005.

19. Programa Nacional de DST e AIDS, Ministério da Saúde. Protocolo para a prevenção da transmissão vertical do HIV e sífilis. Brasília: Ministério da Saúde; 2006.

20. Sousa MGG. Avaliação do grau de implementação do Projeto Nascer maternidades em Pernambuco [Dissertação de Mestrado]. Rio de Janeiro: Escola Nacional de Saúde Pública, Fundação Oswaldo Cruz; 2006.

21. Cavalcante MS, Ramos Júnior NA, Jesus-e-Silva TM, Kerr-Pontes LRS. Transmissão vertical do HIV em Fortaleza: revelando a situação epidemiológica em uma capital do nordeste. Rev Bras Ginecol Obstet 2004; 26:131-8.

22. Feliciano KVO, Kovacs MH. As necessidades comunicacionais das práticas educativas na prevenção da transmissão materno-fetal do HIV. Rev Bras Saúde Matern Infant 2003; 4:393-400.

23. Santos NJS, Buchella CM, Fillipe EV, Bugamelli LG. Mulheres HIV positivas, reprodução e sexualidade. Rev Saúde Pública 2005; 36:12-23.

24. Conselho Federal de Medicina. Resolução no . 1.638, de 10 de julho de 2002. Diário Oficial da União 2002; 9 ago.

25. Basso E, Grazziotim L, Araújo BF, Lorenz DRS. Prevalência de sífilis congênita no Hospital Geral de Caxias do Sul - RS no período de 1998-2002. DST J Bras Doenças Sex Transm 2005; 17:5-9.

26. Schetini J, Ferreira DC, Passos MRL, Salles EB, Santos DDG, Rapozo DCM. Estudo da prevalência de sífilis congênita em um hospital da rede SUS de Niterói - RJ. DST J Bras Doenças Sex Transm 2005; 17:18-23.

27. Melchior R, Nemes MIB, Basso CR, Castanheira ERL, Britto-e-Alves MTS, Buchalla CM, et al. Evaluation of the organizational structure of HIV/AIDS outpatient care in Brazil. Rev Saúde Pública 2006; 40:143-51.

28. Vasconcelos ALR. A epidemiologia na avaliação da qualidade da assistência prestada a gestantes/parturientes portadoras do HIV e seus recém-nascidos [Tese de Doutorado]. Brasília: Universidade de Brasília; 2005.

29. Morimura CR. Situação da testagem anti-HIV em parturientes admitidas em uma maternidadeescola na Cidade do Recife: um estudo transversal [Dissertação de Mestrado]. Recife: Instituto Materno Infantil Prof. Fernando Figueira; 2004. 
30. Benzaken AS, García EG, Sardinha JCG, Dutra Junior JC, Peeling R. Rapid tests for diagnosing syphilis: validation in an STD clinic in the Amazon Region, Brazil. Cad Saúde Pública 2007; 23 Suppl 3: S456-64.

31. Israel GR. "Como é que eu vou dizer para a gestante?" Dilemas morais da oferta do teste anti-HIV a gestantes em trabalho de parto: uma contribuição da bioética [Dissertação de Mestrado]. Rio de Janeiro: Escola Nacional de Saúde Pública, Fundação Oswaldo Cruz; 2002.
32. Vieira AA. Contribuição ao estudo epidemiológico de sífilis congênita no Município de Carapicuíba - SP: ainda uma realidade em 2002. DST J Bras Doenças Sex Transm 2005; 17:10-7.

Recebido em 16/Dez/2008

Versão final reapresentada em 28/Mar/2009 Aprovado em 06/Abr/2009 